\title{
El flash crash y pronostico del S\&P 500 e-mini del 6 de mayo del 2010
}

\section{The crash and forecast flash of the S\&P 500 e-mini of may 6, 2010}

\author{
ROMERO-QUIROZ, Roberto $\dagger^{*}$ \& HERNÁNDEZ-VELEROS, Zeus \\ Universidad Autónoma del Estado de Hidalgo, Maestría en Estudios Económicos \\ ID $1^{\mathrm{er}}$ Autor: Roberto, Romero-Quiroz \\ ID $1^{\mathrm{er}}$ Coautor: Zeus, Hernández-Veleros
}

DOI: $10.35429 / J E D .2019 .20 .6 .1 .5$

Recibido: 10 de Junio, 2019; Aceptado 30 de Septiembre, 2019

\section{Resumen}

En este artículo se describe que es una burbuja financiera y un flash crash, haciendo énfasis en el choque repentino que se originó en los futuros del Standard and Poors E-mini 500 el 6 de Mayo del 2010, el cual dio lugar a grandes pérdidas y a que estos fenómenos obtuvieran más atención por parte del sistema financiero. Posteriormente se pronosticó el S\&P 500 E-mini utilizando la función de transferencia con la finalidad de saber si pudo ser posible haberse anticipado a dicho evento; en el cual se obtuvieron resultados positivos ya que fue posible generar una estimación dicho flash crash.

Choque repentino, Burbuja Financiera, Índice Bursátil, Función de Transferencia, Spoofing

\begin{abstract}
This article describes what is a financial bubble and a flash crash, emphasizing the sudden shock that originated in the futures of the Standard and Poors E-mini 500 on May 6, 2010, which resulted in large losses and that these phenomena get more attention from the financial system. Subsequently, the S\&P 500 E-mini was predicted using the transfer function in order to know if it could be possible to have anticipated said event; in which positive results were obtained since it was possible to generate an estimate said flash crash.
\end{abstract}

Flash Crash, Finnancyal Bubble, Stock Index, Transfer Function, Spoofing 


\section{Introducción}

A lo largo de la historia el sistema financiero ha crecido en una gran magnitud en países desarrollados como subdesarrollados, el cual ha favorecido el crecimiento económico y social. Este sistema se ha destinado en crear diversos instrumentos financieros como las acciones, bonos, commodities, derivados, futuros, entre otros los cuales apoyan a que empresas puedan capitalizarse, solventar deudas o crecer en términos financieros. Hace dos décadas participar en el mercado financiero era muy complicado ya que casas de bolsa o bancos solo permitían grandes cantidades de dinero para poder invertir en algunos instrumentos financieros o simplemente era la falta de conocimiento de las personas. Hoy en día incurrir en este sistema es más sencillo de lo que parece ya que los montos de inversión son más accesibles por tanto la demanda de estos servicios se ha incrementado lo que probablemente ha generado crisis y burbujas financieras.

El siguiente artículo explica que es una burbuja financiera, un flash crash y un pronóstico del S\&P 500 E-mini en el cual se produjo el fenómeno "flash crash"; este estudio se enfoca en realizar un pronóstico el cual beneficiara al inversionista en la toma de decisiones para minimizar el riesgo y maximizar rendimientos

\section{¿Pero que es una burbuja financiera?}

"Una burbuja es un proceso de incremento continuado de los precios de un activo real o financiero por encima del valor fundamental de dicho activo." Cano, 2013.

Cabe mencionar que después del incremento continuo en los precios y llega a un punto máximo que no puede superar dado a la disminución de la demanda, se genera una caída de gran magnitud en los precios lo cual podría generar grandes pérdidas por parte de los inversionistas hasta generar una crisis como la del 2008 también conocida como "La crisis inmobiliaria del 2008" la cual comenzó a principios de los años 90. "A medida que las bajas tasas de interés alimentaban la especulación inmobiliaria, los precios inmobiliarios y las valorizaciones de los constructores de viviendas continuaron aumentando" Robinson, 2018.
Fue a partir del 2005 en donde el rendimiento total de las viviendas comenzó a disminuir hasta alcanzar una caída del $90 \%$ en 2008. Aunque a partir del incremento de permutas de impago por parte de las personas, el mercado ya daba señales sobre una posible burbuja financiera; el sector continuó dando préstamos a tasas muy bajas para viviendas, lo cual ocasionó "La Gran Crisis Financiera" que produjo la mayor perdida en el mercado de valores después de "La Gran Depresión"; debido a la falta de regulación o factores que aún no se han determinado han existido diversas burbujas financieras en la historia, en el artículo que presenta Paul Robinson, 2018 se incluye una de las burbujas más recientes y sonadas en el mercado financiero, "El Bitcoin".

La fiebre por esta criptomoneda inició en el año 2010 la cual cotizaba por menos de un dólar hasta diciembre del 2017 que alcanzó su máximo histórico de $\$ 20,000$ dólares por Bitcoin, cabe mencionar que estas ganancias se produjeron en menos de un año y posteriormente la criptomoneda perdió más del $80 \%$ de su valor.

Estas dinámicas no esperadas son de suma importancia en el análisis de activos financieros ya que estas causan grandes perdidas millonarias por lo cual se han realizado distintos articulos e investigaciones, como es el caso de "Flash Crash: Mueve a los Mercados de Divisas" por Ruth Carson y Michael G Wilson en donde plantean la situación de la moneda japonesa (Yen) a la cual le tomó siete minutos en valorizarse a su nivel mas alto en el cual se había mantenido por casi diez años.

Fue a las 9:30 am hora de Sídney en donde el tipo de cambio japonés se incremento en alrededor del $8 \%$ frente a la moneda australiana la cual según (Ruth Carson, 2019), ha estado en una guerra comercial y ha sido azotada por los incrementos en los tipos de interés por parte de la Reserva Federal de los Estados Unidos.

Algunos analistas creen que este violento movimiento fue causado al momento de que APPLE INC. Anuncio que tendría una perspectiva negativa ante sus ventas, incluso se menciona en el articulo de (Ruth Carson, 2019) que estos "Flash Crash" también pudieron haber sido causados por fallas en los algoritmos del sistema financiero o casas de bolsa. 
Inversionistas con posiciones en corto en el Yen se vieron altamente afectados ya que a pesar de que Japón tuviera cuatro días feriados estos tendrían que pagar para mantener sus posiciones o en dado caso cerrarlas con perdidas. "Fue exacerbado por unas vacaciones en Japón y las ventas minoristas dejan de llenarse en el camino, especialmente en cruces del Yen" dijo Damie Loh, Director de Inversiones del Fondo de Cobertura Ensamble Capital, En Singapur.

Es importante incluir que en el mes de junio y Julio del 2019 este instrumento financiero ha ido recuperando su valor dado al anuncio de la compañía Facebook que próximamente lanzaría una criptomoneda, lo cual generó buenas expectativas para estas monedas. La perspectiva de las criptomonedas como otros instrumentos financieros pueden generan las burbujas las cuales se replican a corto plazo (minutos) denominados "Flash Crash" o choques repentinos.

El cual el siguiente autor lo define como "Un Flash Crash consiste en una caída muy rápida y muy pronunciada del precio de un activo para la que no existe una explicación aparente." (Gonzalo Cabrera López, 2018). Uno de los flash crash mas importantes ocurrió en los futuros del índice S\&P 500 E-mini. Este fue el choque repentino que efectivamente los puso en el mapa, dado lo grande e impactante que fue. El 6 de mayo, el mercado bursátil sufrió un poco de estrés cuando, de repente, los futuros del S\&P 500 E-mini colapsaron en más de un 6\% en aproximadamente siete minutos antes de borrar por completo esas pérdidas en los 10-15 minutos siguientes. En este caso, un comerciante fue acusado y declarado culpable de realizar grandes pedidos de venta que se cancelarían justo antes de que se llenaran. Una estrategia llamada 'spoofing' ${ }^{1}$. Si bien el comerciante fue declarado culpable de causar el flash-crash, estos eventos no suceden de la nada.

Ocurren cuando el mercado ya está en un estado frágil. Cabe mencionar que a la fecha no es posible señalar con cierta eficacia las causas de estos choques repentinos por tanto en el siguiente apartado se mostrará la metodología utilizada para pronosticar un flash crash.

\footnotetext{
${ }^{1}$ Spoofing:" Consiste en introducir de mala fe una orden de venta o de compra siendo conscientes de que no se ejecutará y con la intención de incitar a otros participantes a invertir para más adelante cancelar esas posiciones y sacar partido de ese engaño actuando en el sentido contrario al mostrado en el farol."

(García, 2018)

\section{Metodología}

Función de Transferencia

El objetivo de los modelos de función de transferencia fue buscar concernir dos o más series con cierta temporalidad aplicando modelos de causa que arrojen una predicción. Como menciona Fernández, 2006 en su artículo, este método busca la forma de relacionar una serie temporal la cual se denominada output con otras series que se les llaman inputs.

Fernández (2006) explica que la función de transferencia es de gran utilidad por estas razones:

1. En el modelo lineal, la relación suele ser instantánea y viene establecida a priori.

2. En el modelo lineal general, la relación va de la variable xt a la variable yt, pero la variable yt no influye sobre la variable $\mathrm{xt}$.

3. En el modelo lineal general, la parte de variable respuesta no explicada por la variable independiente $\mathrm{xt}$ es un proceso ut de variables independientes.

"Estos modelos son muy utilizados en todos los campos científicos para evaluar respuestas dinámicas. $S i$ las variables inputs son controlables, estos modelos permiten simular y evaluar políticas alternativas. Si no lo son, ofrecen la posibilidad de estudiar cómo ciertos "escenarios", definidos por posibles evoluciones de la variable explicativa, afectan a la variable respuesta. Además, estos modelos son muy útiles para elaborar predicciones, aunque dependerá del intervalo que se tenga entre observaciones. $\mathrm{Si}$ disponemos de datos anuales (es decir el intervalo entre observaciones es grande), la relación entre la variable explicativa y la respuesta no suele contener retardos y para prever el output es necesario conocer el input". (Fernández, 2006).

Por tanto, esta metodología se aplicó para pronosticar el flash crash en donde los datos utilizados son los precios de los futuros y el índice, los cuales tienen una temporalidad diaria durante tres años desde el 5 de mayo del 2008 al 17 de mayo del 2010. 
$\mathrm{Al}$ aplicar la función de transferencia la variable de entrada es el crudo WTI (C) y el de salida los futuros del S\&P 500 E-mini.(FSP)

\section{Análisis Empírico}

Por la estrecha relación entre las condiciones económicas de Norte América y el precio de crudo, permiten considerar como variable de entrada las cotizaciones del crudo WTI (C), por otra parte, la variable de salida serán las cotizaciones del S\&P 500 E-mini.(FSP), los cuales tienen una temporalidad diaria durante tres años desde el 5 de mayo del 2008 al 17 de mayo del 2010.

El comportamiento de la variable de salida (FSP) relata en la gráfica 1. En este esquema se observa una fuerte caída de 2008 al 2009 , la cual señala la crisis sub-prime. A su vez se presenta una comparativa en el precio histórico de las variables FSP y C en la gráfica 2. En este dibujo se muestra el diferencial en tiempo de las cotizaciones de ambos activos.

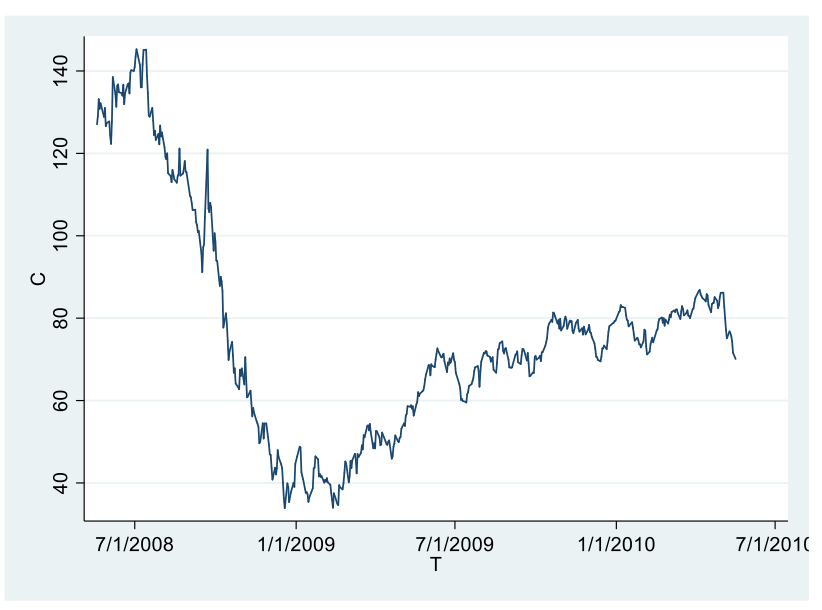

Gráfico1 Cotización histórica del Crudo WTI Fuente: Elaboración Propia

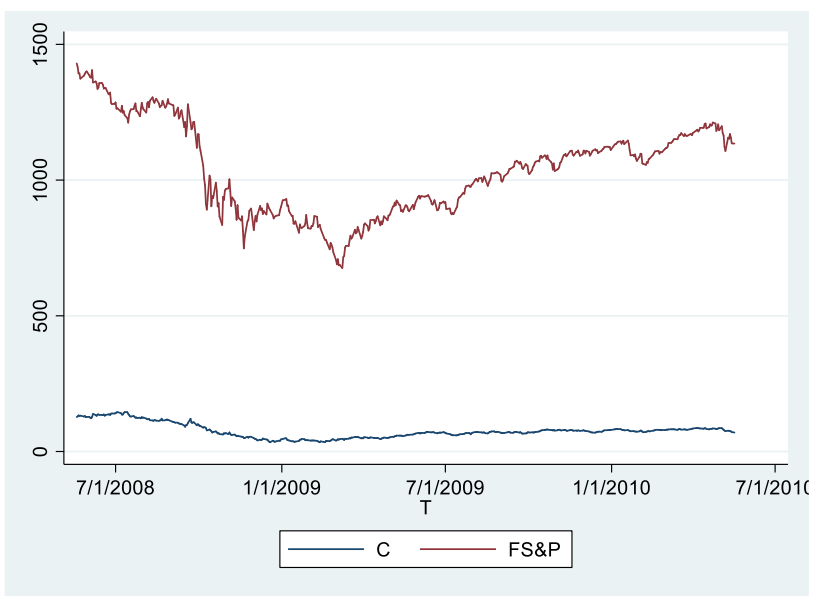

Gráfico2 Comparativa Crudo-S\&P mini Fuente: Elaboración Propia
La metodología propuesta indica como condicional que se rechace la existencia de raíz unitaria en la serie temporal. Para proseguir se asumenen resultados congruentes a la naturaleza de la información requerida.

Se obtuvieron los términos de la función cruzada: Alpha $(\alpha)$ y Beta $(\beta)$, los cuales estan en función del tiempo. La correlación cruzada y las desviaciones estándares de Alpha $\alpha$ y Beta $\beta$, permiten determinar las estimaciones iniciales de la función impulso-respuesta.

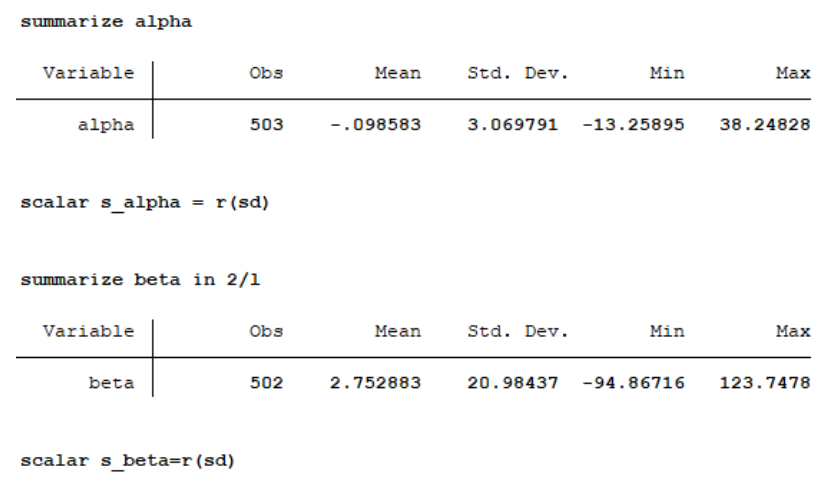

Figura 1 Resulatdo de Alhpa y Beta Fuente: Elaboración Propia

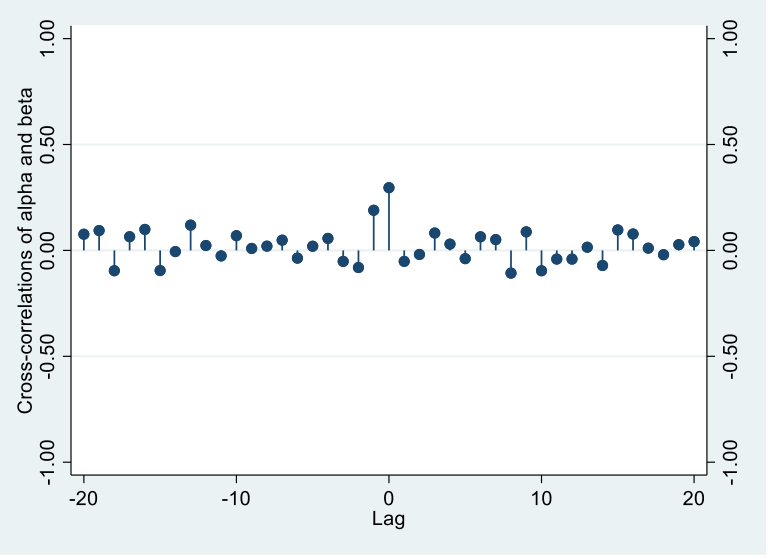

Grafico 3 Correlograma de Alpha y Beta Fuente: Elaboración Propia

La correalción cruzada se utilizó como nueva varaible, en conjunto con las desviaciones estándar de las series de entrada y salida, se filtro para estimaciones iniciales de función impulsorespuest. Con la función de corrleaciones cruzadas se identificó la estructura de retraso en la función impulso-respuesta desde la respuesta impulso. 


\begin{tabular}{|c|c|c|c|}
\hline lag & cross & ir & std \\
\hline 8 & -.1071437 & -.7360268 & -.0890871 \\
\hline 10 & -.0959525 & -.659148 & -.0890871 \\
\hline
\end{tabular}

Figura 2 Resultados de la funación de transferecnia Fuente: Elaboración Propia

Los resultados de la función impulsorespuesta, los cuales se muestran en la figura 2 , permitieron definir los retrasos de la función para proyectar los resultados del FSP:

\begin{tabular}{|c|c|c|c|c|c|c|}
\hline \multicolumn{4}{|c|}{$\begin{array}{l}\text { Sample: } 5 / 29 / 2008-10 / 3 / 2009 \\
\text { Log likelihood }=-2202.721\end{array}$} & \multicolumn{3}{|c|}{$\begin{array}{llr}\text { Number of obs } & = & 493 \\
\text { Wald chi2 (4) } & = & 75093.79 \\
\text { Prob }>\text { chi2 } & =0.0000\end{array}$} \\
\hline $\mathrm{Y}$ & Coef. & $\begin{array}{c}\text { OPG } \\
\text { Std. Err. }\end{array}$ & $z$ & $\mathrm{P}>|z|$ & [95\% Conf. & Interva1] \\
\hline \multicolumn{7}{|l|}{$\mathrm{Y}$} \\
\hline L8. & -.8377407 & .2777503 & -3.02 & 0.003 & -1.382121 & -.29336 \\
\hline L10. & -.7088951 & .2302271 & -3.08 & 0.002 & -1.160132 & -.2576583 \\
\hline _cons & 1297.155 & 154.7035 & 8.38 & 0.000 & 993.9414 & 1600.368 \\
\hline \multicolumn{7}{|l|}{ ARMA } \\
\hline $\begin{array}{l}\text { ar } \\
\mathrm{L} 1 .\end{array}$ & . 9010683 & & 25.89 & 0.000 & .8328668 & .9692698 \\
\hline L2. & .0951418 & .0354402 & 2.68 & 0.007 & .0256803 & .1646033 \\
\hline /sigma & 20.99212 & .3536784 & 59.35 & 0.000 & 20.29892 & 21.68532 \\
\hline
\end{tabular}

confidence interval is truncated at zero.

Figura 3 Resulatdo del modelo ARIMA Fuente: Elaboración Propia

Los resultados de los test de significancia de variables, argumentaron importancia estadistica para los resagos señalados por la función de transferecnia, asi mismo. Por lo cual se realiza la proyección de la varaible de salida, obtenido los que se señalan en el grafico 4.

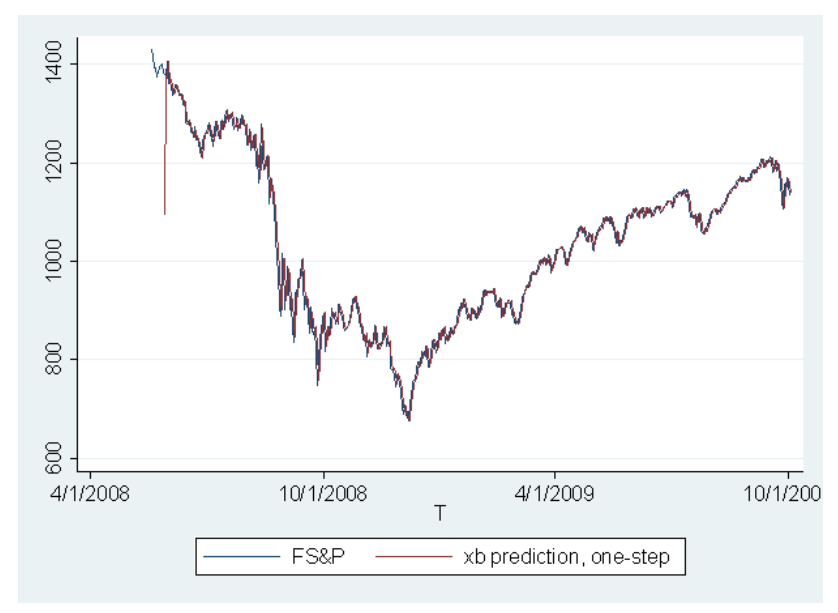

Gráfico 4 Comparativa proyección de S\&P mini y el dato real

Fuente: Elaboración Propia

\section{Conclusión}

El pronóstico presentado en este artículo mostró de forma clara la estimación del flash crash del 6 de mayo del 2010 y que probablemente aplicando la metodología utilizada en este artículo pudo haber sido posible anticiparse ante a ese movimiento. El flash crash ha sido un fenómeno que se ha presentado en distintos instrumentos financieros los cuales se han visto afectados, cabe mencionar que los noticieros económicos y financieros informaron que dicho evento fue motivo de un spoofing, sin embargo no se ha llegado a una explicación que sustente dicho evento y que simplemente se podría hablar de una especulación a corto plazo en los mercados. Es de suma importancia estudiar estas dinámicas no esperadas ya que estas causaron fuertes perdidas a inversionistas y anticiparse a estos eventos disminuiría perdidas y maximizarían ganancias.

\section{Referencias}

Cano, J. S. (2013). Qué es una burbuja financiera? - Métodos de valoración. oro $y$ finanzas.

Fernández, A. d. (2006). Abril.

García, I. (16 de Mayo de 2018). Economía Simple. Obtenido de https://www.economiasimple.net/glosario/spoof ing

Gonzalo Cabrera López. (9 de Junio de 2018). $\mathrm{Mr}$ Finanzas Personales . Obtenido de https://mrfinanzaspersonales.com/que-es-unflash-crash/

Robinson, P. (27 de Diciembre de 2018). Una Breve Historia de las Principales Burbujas Financieras, Crisis y Choques Repentinos.

Ruth Carson, M. G. (2019). Flash Crash, Mueve a los Mercados de Divisas. 6. 